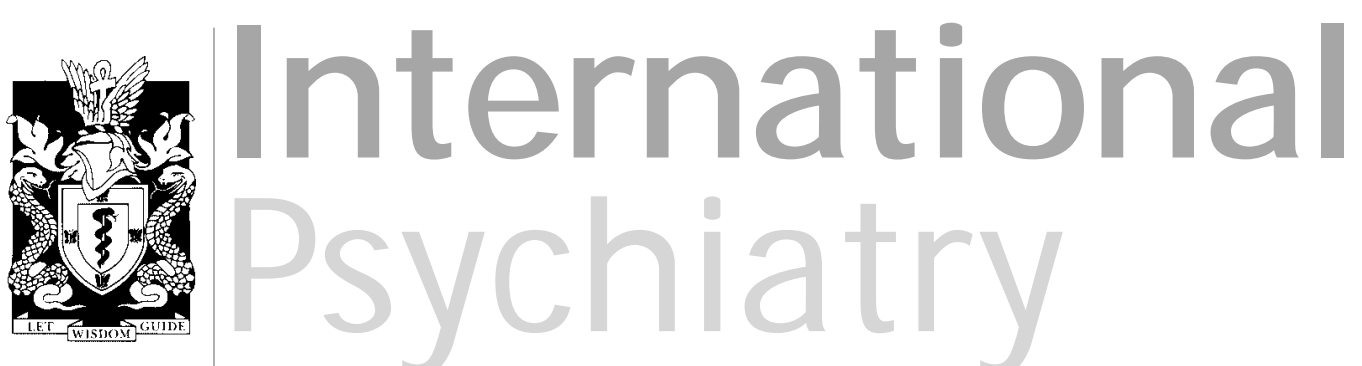

\title{
Mental health research publications from low- and middle-income countries
}

\section{Hamid Ghodse}

Director, Board of International Affairs, and Editor, International Psychiatry

orld-wide, there are more than 471000 psychiatric nurses, 165000 psychiatrists, 147000 psychologists and 229000 social workers operating in the mental health field (World Health O rganization, 2001). It is important that information about recent clinical, service and research developments reaches these professionals and the traditional route for disseminating such knowledge is via professional and scientific journals. H owever, there is clear evidence that the major journals with an international distribution are not accessible to those working in low- and middle-income (LAMI) countries.

There are several contributory factors. First, there is the important issue of affordability. The average psychiatrist working in a develo ping country cannot afford the subscription to even one international journal and, in many countries, very few libraries can afford to subscribe to them either. Journals with a national or regional distribution, produced in the local language, often offer an affordable alternative. Although the quality and originality of their content are variable, so that their role in the dissemination of up-to-date scientific information is doubtful, they do provide a source of information for psychiatrists who do not have access to international journals.

Second, the major international journals (i.e. those with high impact factors) focus heavily on biological psychiatry and psychopharmacology. Most are published in English, but even those clinicians who can understand and read English may find it difficult to comprehend the technical language, concepts and details of increasingly complex and sophisticated research, which anyway appears to have little relevance to the conditions in which they practise.

Unfortunately, the increasing importance attached to impact factors reinforces the trend to carry out research and publish papers on biological psychiatry. This largely excludes researchers from LAMI countries, so that the proportion of articles from non-Western countries in international journals is inversely proportional to the percentage of publications on biological psychiatry topics. The figures speak for themselves:

o The 'rest of the world' (i.e. outside N orth America, Europe and Australia) contributes $6 \%$ of the articles published in the six leading general psychiatry journals (Patel \& Sumathipala, 2001).

o The rate of acceptance of papers from the 'rest of the world' in three leading psychiatry journals is half that for papers from N orth America, Europe and Australia (Patel \& Sumathipala, 2001).

Seen in this context it is unsurprising that LAMI countries do not commit scarce funding to the leading international psychiatric journals: their own contributions are likely to be rejected; the content, written by others, is largely irrelevant to local conditions; and many of the papers are difficult to understand. Further, Saxena et al (2003) found that only four out of a total of 530 editorial and advisory board members of the 10 psychiatric journals with the highest impact factors were based in LAMI countries. It is difficult to escape the conclusion that, currently, these journals are produced by and for mental health professionals in $\mathrm{N}$ orth America, Europe and Australia. They may be international journals but they are surely not global journals.

There are, however, good reasons to promote truly global contributions and readership - not least the enormous unmet mental health needs of LAMI countries, where $85 \%$ of the world's population lives. Teams in all parts of the world can and should contribute to advances in mental health and their findings should be easily accessible to all. More specifically, mental health research should be promoted in LAMI countries in the following areas: advocacy, policy development, and the establishment and expansion of clinical services. There is also a need to educate investigators in research skills. This in turn will contribute to greater international and multicultural understanding of mental health and ill health.
Contents

Editorial

H amid Ghodse 1

Thematic papers -

Cultural variations in the

perception of

psychopathology

INTRODUCED BY

David Skuse

Elizabeth M. Coker 3

L. K. George Hsu 5

Sushrut Jadhav

Country profiles

IN TRODUCED BY

Shekhar Saxena

Atalay Alem -

Ethiopia

Itzhak Levav and

Alexander

Grinshpoon - Israel 10

Anastas Suli, Ledia

Lazëri and Livia

$\mathrm{N}$ ano - Albania

Special paper

Tuviah Zabow

Associations and

collaborations

Benedetto Saraceno 19

N ews, notes, events 22 
Pragmatic action is needed at many levels to close the gap that has developed in professional journals between $\mathrm{N}$ orth America, Europe and Australia and the 'rest of the world'. It would seem sensible, in the first instance, to build on the structure and reputation of the established international journals. First, and immediately, members of their editorial boards with contacts in developing countries should be asked to pass on the message that their journal would welcome submissions. These should be further encouraged by the promise of editorial support and even coaching, with constructive feedback on weak articles and help for those concerned about the standard of their written English. A reputation for welcoming submissions from develo ping countries will encourage others to try. The internet may facilitate this because online submission is so much less costly. Many journals also allow free online access to the world's poorest countries so that authors and their colleagues can see their work online even if they cannot afford a print subscription; this too will encourage further submissions.

More fundamentally, perhaps, the major journals should think carefully about the nature of the research they report, as, indeed, should those who contribute to them. While it is essential to maintain a high standard of scientific merit, not all psychiatric research need be complicated or expensive. Research into mental health services, which can be carried out equally well in LAM I countries as in highly industrialised environments, is as important as the costly high-technology bio logical research. The results and the subsequent papers may in fact be more relevant to clinical practice and therefore more interesting to the majority of clinicians. The publication of papers on service research offers a way of engaging clinicians and of encouraging a genuine and enriching global dialogue - through the enhanced circulation of glo bal journals.

D ifferent approaches to tackling some of these problems were discussed at a meeting in Geneva in $\mathrm{N}$ ovember 2003, organised by the World Health O rganization's D epartment of Mental $\mathrm{H}$ ealth and Substance Abuse. This involved 25 editors representing journals published in low-, middle- and high-income countries; other editors contributed through correspondence and papers. There was a constructive dialogue and a joint statement and catalogue of ideas were agreed (see www.who.int/ mental_health/evidence/en/final_joint_statement.pdf). 0 bvio usly, it will take time to implement the necessary changes and even more time before the consequences of these changes become apparent. H ow ever, this initiative, organised by Drs Saraceno and Saxena of the World $\mathrm{H}$ ealth $\mathrm{O}$ rganization, was an important first step in the right direction and should be commended.

\section{References}

Patel, V. \& Sumathipala, A. (2001) International representation in psychiatric literature. British Journal of Psychiatry, 178, 406409

Saxena, S., Levav, I., Maulik, P., et al (2003) How international are the editorial boards of leading psychiatric journals? Lancet, 361,609

World Health O rganization (2001) Atlas: Mental Health Resources in the World. Geneva: WHO
Dr Brian Martin dale

Dr Shekhar Saxena

Prof. David SKuse

Design ๑ The Royal College of Psychiatrists 2004.

For copyright enquiries, please contact the Royal College of Psychiatrists.

All rights reserved. No part of this publication may be reprinted or reproduced or utilised in any form or by any electronic, mechanical or other means, now known or hereafter invented, including photocopying and recording, or in any information storage or retrieval system, without permission in writing from the publishers.

The views presented in this publication do not necessarily reflect those of the Royal College of Psychiatrists, and the publishers are not responsible

for any error of omission or fact.

The Royal College of Psychiatrists is a registered charity (no. 228636)

Printed in the UK by Henry Ling Limited at the Dorset Press, D orchester DT1 1HD.

\section{Cultural variations in the perception of psychopathology}

\section{David Skuse}

Behavioural and Brain Sciences Unit, Institute of Child Health, London WC1 1EH, UK, email d.skuse@ich.ucl.ac.uk

or many years now there has been debate among psychiatrists concerning the specificity of diagnostic practices to particular cultures. The harmonisation of criteria for diagnostic practice, outside $\mathrm{N}$ orth America, has been one of the great achievements of the World Health $O$ rganization. $\mathrm{N}$ ow, there seems to be a resurgence of interest in how culturally bound our ideas of psychopathology are, or should be.

In an article entitled "H ow "culture bound" is "cultural psychiatry"?', Sushrut Jadhav draws the conclusion that cultural psychiatry is becoming a specialty in its own right, and provocatively challenges us to consider the possibility that this is yet another opportunity for academics to indulge their intellectual curiosity. We can detect a theme here, which is reflected in the call to action by George $\mathrm{H}$ su: the way forward for international psychiatry is not simply to export models of psychiatry developed in the industrialised West to parts of the world where they have little direct relevance to the mental health needs of the majority. Is the profession in the UK guilty of training psychiatrists from overseas in its own image of this complex discipline, and thereby effectively laying the foundations for an ability to 'poach' them in due course, to fill the ever-expanding need for mental health professionals?

Jadhav makes the point that, in developing countries, which have a different perspective on mental distress and disorder to the $\mathrm{N}$ orth American/European one, terms like 'life events' may have quite distinct connotations. He draws our attention to the possibility that we have much to learn from anthropologically informed methods of enquiry. An eloquent example of such an enquiry comes from Elizabeth 